\title{
Microsatellite frequencies vary with body mass and body temperature in mammals, suggesting correlated variation in mutation rate
}

Substitution rate is often found to correlate with life history traits such as body mass, a predictor of population size and longevity, and body temperature. The underlying mechanism is unclear but most models invoke either natural selection or factors such as generation length that change the number of mutation opportunities per unit time. Here we use published genome sequences from 69 mammals to ask whether life history traits impact another form of genetic mutation, the high rates of predominantly neutral slippage in microsatellites. We find that the length-frequency distributions of three common dinucleotide motifs differ greatly between even closely related species. These frequency differences correlate with body mass and body temperature and can be used to predict the phenotype of an unknown species. Importantly, different length microsatellites show complicated patterns of excess or deficit that cannot be explained by a simple model where species with short generation lengths have experienced more mutations. Instead, the patterns probably require changes in mutation rate that impact alleles of different length to different extents. Body temperature plausibly influences mutation rate by modulating the propensity for slippage. Existing hypotheses struggle to account for a link between body mass and mutation rate. However, body mass correlates inversely with population size, which in turn predicts heterozygosity. We suggest that heterozygote instability, $\mathrm{HI}$, the idea that heterozygous sites show increased mutability, could provide a plausible link between body mass and mutation rate. 
1 Microsatellite frequencies vary with body mass and body 2 temperature in mammals, suggesting correlated variation 3 in mutation rate.

4 William $\operatorname{Amos}^{1} \&$ Laura N. S. Filipe

$5{ }^{1}$ Department of Zoology, Downing Street, Cambridge, CB2 3EJ, UK

6 'Hills Road Sixth Form College, Cambridge, CB23 8PE, UK

7 Corresponding author: W. Amos, email: w.amos@zoo.cam.ac.uk

8 Department of Zoology, Downing Street, Cambridge, CB2 3EJ, UK

9 phone +441223336616 
11 Classical studies of molecular evolution often refer to the 'molecular clock'

12 (Zuckerkandl, Pauling 1962; Margoliash 1963), the notion that genetic

13 mutations occur randomly and at constant rate such that the degree of

14 divergence seen between species tends to be proportional to geological time.

15 However, even from its early days, the constancy of the clock has been

16 questioned (Fitch 1983). Among many deviations, one of the most intriguing

17 is the way the rate of molecular evolution appears to covary with life history

18 traits (Martin, Palumbi 1993; Smith, Donoghue 2008; Lanfaer, Welch,

19 Bromham 2010; Bromham 2011). A good example is body mass in

20 mammals, where larger species evolve more slowly than smaller species

21 (Bromham, Rambaut, Harvey 1996; Lartillot, Poujol 2011). The most widely

22 accepted explanation for this involves a link to generation length, but fit to a

23 simple model is poor, in that a doubling of generation time on average results

24 in much less than a halving of evolutionary rate (Welch, Bininda-Emonds,

25 Bromham 2008).

26 Despite many studies exploring the relationship between life history traits

27 and evolutionary rate, the overwhelming majority have focused on either

28 mitochondrial DNA or nuclear 'genes'. Few, if any, have looked explicitly at

29 the fastest evolving components of the genome such as short tandem

30 repeats (STR), yet it is here that the largest effects might be expected.

31 Microsatellites form an important class of STR with repeats one to six

32 nucleotides in length and occur abundantly in higher organisms (Bruford,

33 Wayne 1993). Most mutations involve molecular slippage causing the gain or

34 loss of repeat units (Schlötterer, Tautz 1992), often with bias favouring

35 contraction or expansion (Dermitzakis et al. 1998; Ellegren 2000; Xu et al.

36 2000). Mutation rate increases with repeat number in a strongly non-linear

37 fashion (Weber 1990; Kelkar et al. 2008). Maximum repeat number of a

38 given microsatellite motif varies between species (Di Rienzo et al. 1994),

39 probably due to a thermally-influenced upper length boundary (Amos, Clarke

40 2008).

41 Microsatellites seem to be 'born' when point mutations by chance create

42 sufficient consecutive repeats for slippage to occur (Messier, Li, Stewart

43 1996), persist for a finite time and eventually 'die' (Taylor, Durkin, Breden

44 1999). The process of death is poorly understood and may well vary between

45 loci, some being deleted while others degenerate through substitutions

46 within the repeat array. However, regardless of the exact details, the relative

47 frequency of any given length of microsatellite in the genome should tend to

48 reflect the relative time spent by an average microsatellite during its lifetime 
49 at that length. Short microsatellites are common both because all

50 microsatellites are short at some point and because their low mutation rate

51 causes them to linger at this length. Conversely, long microsatellites are rare

52 both because many loci never become long and because, once a locus

53 becomes long, the much higher mutation rate causes it rapidly either to

54 shrink or to 'die'. Consequently, the length-frequency distribution of

55 microsatellites in the genome can be viewed as a form of standing wave with

56 microsatellite birth acting as a source and microsatellite death a sink. The

57 exact shape of this wave will reflect a complex interplay between the rates of

58 birth and death and the relationships between repeat number, mutation rate

59 and mutation bias.

60 Differences between species in the shape of the standing wave may allow a 61 distinction to be made between two key hypotheses concerning life history

62 traits. In the generation length hypothesis, variation in mutation rate arises

63 from differences in the number of mutation opportunities per unit time. We

64 call these mutation number models. In contrast, factors such as metabolic

65 rate or body temperature (Tuntiwechapikul, Satazar 2002) may impact

66 mutation rate directly by changing the probability that a mutation occurs in

67 any given round of DNA replication. We call these mutation rate models. For

68 base substitutions, the two models yield identical predictions in terms of

69 sequence divergence. However, the same does not necessarily hold for

70 microsatellites.

71 If the system is at or very close to equilibrium the shape of the standing wave

72 will be stable and should not change in response to more or fewer mutations

73 occurring when generation length shortens or lengthens respectively. The

74 shape of the standing wave should only change in response to changes in the

75 parameters that influence its shape, specifically if the relative mutability of

76 different length microsatellites changes or if the relationship between

77 mutation bias and repeat number changes. For example, if longer

78 microsatellites become relatively more mutable, an average microsatellite

79 will spend less time 'long', and a new equilibrium wave will be created in

80 which the frequency of long microsatellites is reduced. Such changes should

81 not occur under mutation number models but are a possible (though not

82 necessary) consequence of mutation rate models.

83 To help understand the relationship between life history traits and mutation

84 rate we used mammalian genome sequences to determine length-frequency

85 profiles for three common microsatellite motifs. We then asked: (a) whether

86 these profiles differ between species and (b) whether any differences relate

87 to two key phenotypes, body temperature or body mass (Gillooly et al. 2005). 
89

90 We downloaded 69 complete or largely complete mammalian genomes from

91

92

93

94

95

96

97

98

99

100

101

102

103

104

105

106

107

108

109

110

111

112

113

114

115

116

117

118

119

120

122

123

124

125 the NCBI and UCSC websites (http://www.ncbi.nlm.nih.gov/, http://genome.ucsc.edu/), some as separate assembled chromosomes and others as bulk files of larger, unplaced contigs. Given the different formats, and for complete control, we counted microsatellites using custom $\mathrm{C}++$ scripts written by WA, focusing on three motifs: ' $A C$ ', ' $A T$ ' and ' $A G$ '. Together with their reverses ('CA', 'GA', 'TA'), complements ('TG', 'TC') and their reverse complements ('GT', 'CT') these embrace all possible dinucleotides with the exception of ' $C G$ ' and ' $G C$ ', which are extremely rare due to the high mutability of CpG (Kelkar et al. 2008). In fact, we did also look at (CG) repeats but their general rarity precluded a meaningful analysis: tracts carrying nine or more repeats were absent from at least some species and human $(\mathrm{CG})_{3}$ is 30 fold rarer than $(\mathrm{AT})_{3}$. A lower limit of four repeats was set to reflect the shortest length at which slippage is likely important. An upper limit of 30 repeats was set because longer tracts are extremely scarce in some species, probably due to a combination of the known dependence on body temperature and biases arising from differences between sequencing platforms. We also looked at three triplet repeats, 'ATT', 'AGG' and 'AAC'. Substitutions within the repeat tract occur at an appreciable rate and act to reduce the rate of slippage (Џin et al. 1996).

Since a minority of tracts are interrupted and interrupted tracts seem to mutate approximately as if they comprise just the longest pure tract (Weber 1990), we began by counting only pure repeat tracts. No minimum separation was enforced between tracts, such that $(A C)_{10} A T(A C)_{4}$ would count one $(A C)_{10}$ and one $(A C)_{4}$ : the longer tract would count anyway while the shorter tract would add negligibly to the very large number of isolated $(A C)_{4}$ tracts. To explore the possible impact of interruption mutations we then repeated the analysis using tracts of the form $(X Y)_{a} Z Z(X Y)_{b}$, where $X Y$ is the target motif, $Z Z$ represents any pair of bases that is not the target motif. We required $a$ and $b$ both to be greater than one and that at least one was three or greater.

\section{Statistical analysis}

All analyses were conducted using R 2.15.2 (http://cran.r-project.org/). To control for shared ancestry, we used the method of phylogenetically independent contrasts (PICs) (Felsenstein 1985; Purvis, Rambaut 1995), implemented in the package 'ape' (Paradis, Claude, Strimmer 2004). 
126 Calculation of PICs requires a phylogeny with associated branch lengths

127 (Figure S1). Wherever possible, including all deeper nodes, dates were read

128 from Figure 1 in Meredith et al. (Meredith et al. 2011). Dates for other nodes

129 were extracted from individual group phylogenies (Fernandez, Vrba 2005;

130 Osada et al. 2008; Agnarsson et al. 2011; Perelman et al. 2011; Fabre et al.

131 2012) (for the resulting phylogeny, see Figure S1).

132 To test whether microsatellite frequency differences correlate with life history

133 characteristics we focused on two phenotypes, body temperature and sex-

134 averaged adult body mass. Body temperatures vary with factors such as

135 exercise and digestion, and can fall dramatically during hibernation or torpor.

136 We therefore sought values for the 'normal' active temperature, and for

137 consistency take as many as possible measurements from one large study by

138 Clarke and Rothery (Clarke, Rothery 2008) which also gives body masses

139 (Table S1). For four temperatures and one mass we were unable to find

140 reliable values and had to use educated guesses based on closely related

141 species (for details, see Table S2). For each repeat number (range four to 30)

142 in each of the three dinucleotide motifs (AC, AG, AT) we fitted multiple linear

143 regressions with $\log _{e}$ (frequency) as the response and body temperature $\left({ }^{\circ} \mathrm{C}\right)$,

$144 \log _{10}$ (body mass, $\mathrm{Kg}$ ) and \%GC content as predictors. \%GC content was

145 included to control for the possibility that this influences microsatellite

146 frequencies and has a small but marginally significant impact on the

147 frequencies of the very shortest microsatellites (repeat number $<10$ ). All

148 variables were initially (see below) included as 68 PICs. Once adequate

149 model fit had been achieved, significance was determined using the

150 command 'anova' to compare models with and without the term of interest

151 being fitted. Thus, if the full model is written $F \sim P 1+P 2+G C(F=\log$

152 frequency, $\mathrm{P} 1=$ phenotype1, $\mathrm{P} 2=$ phenotype2, $\mathrm{GC}=\% \mathrm{GC}$ ), the significance of

153 P1 would be assessed by comparing the full model with the model $F \sim P 2+$

154 GC.

155 Model fit was explored using the command 'plot', with particular attention

156 given to models yielding high significance. In most cases the residuals were

157 reasonably normally distributed and there were no major outliers or points

158 with high leverage. However, in some of the PIC models one data point, that

159 corresponding to the deep node separating rodents and primates, appeared

160 as a big outlier across all motifs and was excluded from all analyses. Two

161 other deep nodes, primate-Eulipotyphla and Eulipotyphla-carnivore, were

162 medium outliers for some motifs but other others and were excluded from all

163 models of the motifs where they were a problem. 
165

166

167

168

169

170

(a) Do closely related species differ in their microsatellite frequencies? We began by asking whether closely related species exhibit potentially informative differences in their microsatellite frequencies, based on the wellcurated genomes of six higher primates (human, chimpanzee, bonobo, gorilla, orang-utan and gibbon). The frequency of any given species microsatellite length - motif combination, $F_{i j k}$, was expressed on a log scale:

$$
F_{i j k}=\log _{e}\left(\frac{m_{i j k}+1}{n_{i}} \dot{\dot{j}}\right)
$$

171 where $m_{i j k}$ is the raw count of that microsatellite motif $k$, length $j$ in species $i$ 172 and $n_{i}$ is the number of nucleotide bases assayed in the genome of species $i$.

173 To create profiles depicting the relative frequencies of each microsatellite in 174 each species across the range of lengths considered (hereafter referred to as

175 'profiles'), individual frequencies were expressed as deviations from the

176 mean of all six species (i.e. $F_{i j k}-\overline{F_{j k}}$ ). Since higher primates have rather

177 similar generation lengths (Langergraber et al. 2012) and for general

178 comparison we repeated this analysis on a second group, the Afrotheria, 179 which have an exceptionally wide range of generation lengths, from $\sim 2$ years 180 for the Cape elephant shrew up to 25 years for the African elephant (Pacifici 181 et al. 2013).

182 The resulting profiles for primates reveal obvious and rather complicated 183 differences between species (Figures 1 and 2) that increase in amplitude with 184 repeat number, probably reflecting the higher mutation rates of longer 185 microsatellites (Kelkar et al. 2008), and appear more extreme for AT motifs 186 where the rate of slippage is higher (Schlötterer, Tautz 1992). Even profiles

187 from the closest relatives, the bonobo (blue) and chimpanzee (red), are easily 188 distinguished, implying shape changes that can occur over timescales as 189 short as a million years. Large profile differences might arise from 190 differences between sequencing platforms, depth of coverage and state of 191 assembly. For example, long microsatellites might be rare in genomes 192 assembled from short reads. However, this is not what we see. Species with 193 few long microsatellites at one motif usually show excesses either of long 194 microsatellites of the other motif or of medium long microsatellites of the 195 same motif. More generally, most profiles recall pulses moving through the 196 length-frequency distribution, with excesses at one length balanced by 197 deficits at another and, for any given species, the peaks and troughs tending 198 to occur at different repeat numbers in the two motifs shown. Profiles for the 
199

200

201

202

203

204

205

206

207

208

209

210

211

212

213

214

215

216

217

218

219

220

221

222

223

224

225

226

227

228

229

230

231

232

233

234

235

236

237

238

Afrotheria (Figures 3 and 4) show broadly similar features, though with generally greater amplitude due to the larger evolutionary distances between species. As with the primates, rank order of species changes constantly with repeat number and profiles for a given species often differ dramatically between AT and AC.

(b) Do microsatellite frequency differences correlate with phenotype? To test for links to life history traits we fitted multiple regressions with phylogenetic correction (see methods). Associations are summarised as estimates of the slope, to give the size and direction of any relationship, with associated $95 \%$ confidence intervals to allow significance to be judged (Figures 5 and 6, for raw data see Table S2, for triplet repeat plots see Figures $\mathrm{S} 2$ and S3). For comparison we also present the same analysis for microsatellites carrying one interruption (Figures 7 and 8). The plots for interrupted tracts are generally very similar to those of equivalent pure tracts, presumably because most interrupted tracts derive rather recently from a pure tract of very similar length. Supporting previous observations that microsatellite mutation rate increases with repeat number, trend strength tends to increase with repeat number (Weber 1990; Kelkar et al. 2008; Sun et al. 2012). A general lack of significant trends among the shortest microsatellites could reflect either the lack of causal link or the mutation rate of short microsatellites, which may be too low to track phenotype evolution in mammals. Note, in interpreting these graphs it is important not to forget the temporal element. The slope estimates are based on PICs and therefore on average tend to reflect how short-term changes in phenotype covary with short-term changes in microsatellite frequency. Over longer periods the observed trends may be different and possibly even reverse.

Overall significance of the trends is difficult to assess due to the need to control jointly for both 162 multiple tests ( $=27$ lengths $\times 3$ motifs $\times 2$ phenotypes) and some unknown level of autocorrelation between similar length microsatellites. Nonetheless, there are two reasons to believe the trends are genuine. First, many individual slopes differ significantly either from zero or from each other. Second, although all six profiles exhibit broadly similar patterns, wherein slope is constant or increasing up to a point of inflexion after which it becomes increasingly negative, there are differences between phenotype. The slopes for mass and temperature tend to be negatively correlated (Figures S4 - S6). Thus, the effect of increasing body temperature tends to be similar to that of decreasing body size and hence increasing population size, consistent with higher mutation rates in larger populations. 
239 (c) Can phenotype be predicted from the frequencies of different length

240 microsatellites?

241 As indicated, overall significance is difficult to determine due to non-

242 independence. Moreover, the assumption of Brownian evolution that

243 underpins the PIC analysis is unlikely to be met. Consequently, we sought an

244 overall view of the informativeness of these profiles through a form of cross-

245 validation. Each species was assigned to one of 13 major clades, each

246 separated from all other species by at least 50 million years: Artiodactyls

$247(n=8)$; Cetaceans $(n=2)$; Perissodactyls $(n=2)$; Chiroptera $(n=6)$; Carnivores

$248(n=6)$; Eulipotyphla $(n=3)$; Primates $(n=14)$; Glires $(n=14)$; Tree shrews

$249(n=1)$; Afrotheria $(n=7)$; Xenarthra $(n=2)$; Marsupials $(n=3)$; Monotremes

$250(n=1)$. Note, in view of the unusual ecology of Cetaceans, Cetartiodactyls

251 were divided into the two antiquated constituent groupings.

252 Body mass and body temperature were then estimated for each species in 253 turn, using simple linear regressions with no phylogenetic correction, based 254 only on data from the 12 other clades. Specifically, multiple regressions were 255 constructed with the phenotype of interest as the response variable with 256 predictor variables microsatellite frequency, $F$, defined above and the other 257 phenotype. Regressions were fitted for all combinations of repeat type $(\mathrm{N}=3)$ 258 and repeat number (range 4 to 30 ). Wherever a significant regression 259 ( $P<=0.01$, for other thresholds see Table S3) was obtained, the R function 260 'predict' was used to obtain an estimate of the response phenotype for the 261 focal taxon. Non-significant regressions were not used since these will 262 largely contribute only noise. Predicted phenotypes were then taken as the 263 average of all individual estimates across all repeat numbers and repeat 264 types. Microsatellite length frequency profiles have significant predictive 265 power for both body temperature $\left(r^{2}=12 \%, P=0.004\right)$ and body mass (Figure $2669, r^{2}=38 \%, N=68, P=1.5 \times 10^{-8}, r^{2}$ rising to $43 \%$ if the killer whale is excluded).

267 Given that estimates of mass vary between databases, particularly for 268 strongly sexually dimorphic species, we tested the robustness of our analysis 269 repeating it twice, each time randomly either doubling or halving each 270 species' mass. The resulting r-squared values of $41 \%$ and $36 \%$ indicate the 271 six orders of magnitude of mass covered by our species range completely

272 dominates and biologically plausible variation in individual species estimates.

\section{Discussion}

274 Here we test the hypothesis that the widely reported link between

275 substitution rate and life history traits extends to neutral, rapidly evolving

276 microsatellites. We find that the frequencies of different length microsatellites 
277 vary greatly even between closely related species, and that these differences 278 correlate with both body temperature and body mass. Our results support a 279 link between evolutionary rate and life history traits but the patterns we find 280 are difficult to explain by a simple model where larger species evolve more 281 slowly due to longer generation length.

282 The complicated patterns of frequency differences seen in Figure 1 show that 283 models based on mutation number are inadequate. A key feature of

284 mutation number models is that rank order frequency is expected to be 285 constant (or the inverse) across all motif-length combinations: a species' rank 286 depending only on the number of generations they have been through since 287 the common ancestor of mammals. Constant rank order is also expected if 288 species differences arise mainly due to differences between sequencing 289 platforms, with some species carrying fewer long microsatellites across all 290 motifs. That rank order varies greatly (54 different orders for the six primates in 81 length-motif combinations) therefore suggests no more than a minor role for generation length and argues against large sequencing

293 platform biases. Similar results are found for the Afrotheria. The relatively minor impact of sequencing platform and assembly state is also indicated by the correlations we find between microsatellite frequency and phenotype. Indeed, since differences due to sequencing platform almost certainly exist

298 and would inflate the error variance, our reported results most likely

299

300 underestimate the true effect sizes.

301

302

303

304

305

306

307

308

309

310

311

312

313

314

315

316

317
Apart from generation length, three other models have been proposed for how mass and substitution rate might be correlated (Bromham 2011). First, mass might exert an influence through a correlation with metabolic rate and / or body temperature. However, we show an independent effect of body mass in models where body temperature is also fitted. Moreover, body mass and body temperature are only weakly correlated $\left(r^{2}=0.031, n=596\right.$, data from Clarke \& Rothery (Clarke, Rothery 2008)). Second, mutation rate should evolve to increase with a species' need to gamble (Giraud et al. 2001). Larger species produce fewer, individually costlier offspring so may favour less gambling and therefore lower mutation rates. This explanation fails because microsatellites are mostly neutral and evolve primarily through slippage (Schlötterer, Tautz 1992), so microsatellites unlikely to be affected by evolved changes in substitution rate, should these exist and evolve at a high enough rate in higher vertebrates. Microsatellite mutation rates might vary rapidly through evolved changes in the mismatch repair system, particularly if a selfish evolutionary arms race were involved. However, this model fails through lack of any plausible link to phenotype: selfish processes tend to be 'blind'. 
318 The last possibility relates to smaller species tending to have both higher 319 fecundity and larger population sizes (Damuth 1981). Genetic drift operates 320 more slowly in large populations, thereby allowing a higher proportion of 321 mildly deleterious mutations to be removed by selection (Ohta 1987). This

322 model also founders on two key elements. Most importantly, the nearly 323 neutral theory predicts slower evolution in larger populations, the exact 324 opposite of what has been observed (Amos 2011). Our current data agree: in

325 Figure 2 the slopes for the two phenotypes are generally negatively 326 correlated (Figure S3), suggesting that an increase in body temperature (= 327 more slippage) has a similar impact to a decrease in body size. There is also 328 the recurrent problem with models that invoke selection that, while some 329 microsatellites occur in functional regions and may be selected (The 330 Huntington's Disease Collaborative Research Group 1993), most are likely 331 neutral (Buschiazzo, Gemmell 2010) or effectively so.

332 One model not previously considered for how microsatellite frequencies and 333 body mass might become correlated is heterozygote instability $(\mathrm{HI})$. The $\mathrm{HI}$ 334 hypothesis proposes that recognition and 'repair' of heterozygous sites 335 during meiosis leads to additional rounds of DNA replication that in turn 336 provide opportunities for extra mutations (Amos 2010b). HI predicts that 337 mutation rate should scale with population size and hence with body mass due to the classical positive relationship between heterozygosity and population size (Hartl 1988; Slatkin 1995). Evidence for HI comes variously from the way SNPs are clustered, which fits well with a model where new mutations occur preferentially near pre-existing polymorphisms (Drake 2007; Amos 2010a; Schrider, Hourmozdi, Hahn 2011), from the reduction in mutation rate that occurred as humans lost variability leaving Africa (Amos 2013) and, most directly, from the correlation between microsatellite mutation rate and modern human population size (Amos 2011). Direct mutation counting gives conflicting results, two studies providing support for HI (Amos et al. 1996; Masters et al. 2011) but another not (Sun et al. 2012), though the latter appears not to control for the greater detectability of mutations in homozygotes. While $\mathrm{HI}$ remains an unproven hypothesis, it has the potential to explain some aspects of our results that cannot easily be accommodated easily by existing models. First, $\mathrm{HI}$ acts directly on mutation rate rather than mutation number, so could change the shape of a microsatellite length-frequency profile. Second, HI can explain mutation rate differences between morphologically and physiologically similar relatives such as chimpanzees and bonobos, as long as they differ in their demographic histories. Third, $\mathrm{HI}$ 
357 can account for links to life history traits for both base substitutions (Amos

358 2013) and slippage in microsatellites (Amos 2011), and operates

359 independently of natural selection. Finally, the impact of body mass is

360 consistent with predictions from $\mathrm{HI}$ because, as discussed above, higher rates

361 of slippage seem linked to greater population size (=smaller mass). Despite

362 this, $\mathrm{HI}$ does not offer a complete explanation because it cannot account for

363 links between life history traits and haploid regions of the genome such as

364 mitochondrial DNA (Samuels 2004; Khaidakov, Siegel, Shmookler Reis 2006;

365 Welch, Bininda-Emonds, Bromham 2008; Nabholz, Glémin, Galtier 2009).

366 Comparing the ways microsatellite frequencies correlate with body mass and

367 body temperature reveals an apparent contradiction. While the largest and

368 most significant effect sizes are found for body temperature, the greatest

369 predictive power is for body mass. The answer probably lies with the

370 timescales over which the two traits vary. Body temperature can go up as

371 well as down and may well change rapidly, for example if a species evolves

372 hibernation. Such patterns are captured effectively by independent

373 contrasts, a method that focuses on trait changes relative to a (usually

374 recent) common ancestor. By comparison, body mass tends to be much

375 more stable, varying much more between than within major clades and most

376 trends are towards larger body size (Alroy 1998). For mass, therefore,

377 independent contrasts often embrace rather little change and tend to be

378 relatively uninformative, while regressions across all taxa cover a wide range

379 of sizes and are robust enough to give excellent predictability.

380 Mutation bias seems an integral feature of microsatellite evolution (Primmer 381 et al. 1996; Sun et al. 2012) and may obey complicated rules (Ellegren 2000;

382 Xu et al. 2000). Our results also support the existence of biases. Without

383 bias, the shape of the allele length-frequency profile will be stable because

384 the average change in length of an allele over time is zero, regardless of

385 variation in either mutation rate or mutation number. Shape changes require 386 the joint action of mutation bias, to create asymmetry, and a change to the 387 relative mutability of different length alleles. For example, suppose short 388 microsatellites tend to expand under an upward mutation bias (Xu et al. 3892000 ) and that a change to the mutation process either creates more short 390 microsatellites or increases their relative mutability. The result would be a 391 pulse of excess frequency that would migrate upwards in length until lost at 392 the upper boundary. Such a model is speculative, and more modelling is 393 needed to establish its viability, but it seems intuitively in keeping with the 394 patterns seen in Figures 1 - 4. 
395 The ability to predict a species' body mass to about an order of magnitude 396 may appear unremarkable but we argue that this predictability can be seen 397 as surprisingly good. First, mass is unlikely to influence microsatellite 398 frequencies directly but instead must act through a (likely weak) correlation 399 with some other factor. Second, even the high mutation rates of 400 microsatellites are unlikely to prevent appreciable lag between the shape of 401 the length-frequency distribution and changes to phenotype. Third, we 402 consider only two factors in our prediction model, body mass and body 403 temperature. If other factors are important, their omission will degrade 404 predictive power. All these elements will inflate the error variance and 405 suggest that more detailed modelling could allow an appreciable increase in 406 predictability.

407 In conclusion, the relative frequencies of microsatellites of different lengths 408 vary markedly between different species of mammal. We show how these 409 differences are not random but instead correlate with two phenotypic traits, 410 both of which plausibly influence microsatellite mutation rate. Even with 411 crude measures of phenotype, the length frequency profiles contain enough 412 information to make meaningful inferences about an unknown species.

413 Although of little use on its own, the implication is that future research aimed 414 at understanding and modelling the impact of genome-wide changes in 415 mutation rate has the potential to open a window on aspects of historical 416 ecology that were previously beyond our reach.

\section{References}

418

419

420

421

422

423

424

425

426

427

428

429

430

431

432

433

434
Agnarsson, I, CM Zambrana-Torrelio, NP Flores-Saldana, LJ May-Collado. 2011. A time-calibrated species-level phylogeny of bats (Chiroptera, Mammalia). PLoS currents 3:RRN1212-RRN1212.

Alroy, J. 1998. Cope's rule and the dynamics of body mass evolution in North American fossil mammals. Science 280:731-734.

Amos, W. 2010a. Even small SNP clusters are non-randomly distributed: is this evidence of mutational non-independence? Proc. R. Soc. B 277:1443-1449.

Amos, W. 2010b. Heterozygosity and mutation rate: evidence for an interaction and its implications. BioEssays 32:82-90.

Amos, W. 2011. Population-specific links between heterozygosity and the rate of human microsatellite evolution J. Mol. Evol. 72:215-221.

Amos, W. 2013. Variation in heterozygosity predicts variation in human substitution rates between populations, individuals and genomic regions. PLoS ONE 8:e63048.

Amos, W, A Clarke. 2008. Body temperature predicts maximum microsatellite length in mammals. Biol. Lett. 4:399-401. 
435

436

437

438

439

440

441

442

443

444

445

446

447

448

449

450

451

452

453

454

455

456

457

458

459

460

461

462

463

464

465

466

467

468

469

470

471

472

473

474

475

476

477

478

479

480

481

Amos, W, SJ Sawcer, R Feakes, DC Rubinsztein. 1996. Microsatellites show mutational bias and heterozygote instability. Nature Genetics 13:390391.

Bromham, L. 2011. The genome as a life-history character: why rate of molecular evolution varies between species Phil. Trans. R. Soc. Lond. B 366:2503-2513.

Bromham, L, A Rambaut, PH Harvey. 1996. Determinants of rate variation in mammalian DNA sequence evolution. J. Mol. Evol. 45:610-621.

Bruford, MW, RK Wayne. 1993. Microsatellites and their application to population genetic studies. Current Opinion in Genetics and Development 3:939-943.

Buschiazzo, E, NJ Gemmell. 2010. Conservation of human microsatellites across 450 million years of evolution. Genome Biol. Evol. 2.

Clarke, A, P Rothery. 2008. Scaling of body temperature in mammals and birds. Functional Ecol. 22:58-67.

Damuth, J. 1981. Population density and body size in mammals. Nature 290:699-700.

Dermitzakis, ET, AG Clark, C Batargias, A Magoulas, E Zouros. 1998. Negative covariance suggests mutation bias in a two-locus microsatellite system in the fish Sparus aurata. Genetics 150:1567-1575.

Di Rienzo, A, AC Peterson, JC Garza, AM Valdes, M Slatkin. 1994. Mutational processes of simple sequence repeat loci in human populations. Proc. Natl. Acad. Sci. USA 91:3166-3170.

Drake, JW. 2007. Too many mutants with multiple mutations. Crit. Rev. Biochem. Mol. Biol. 42:247-258.

Ellegren, H. 2000. Heterogeneous mutation processes in human microsatellites. Nat. Genet. 24:400-402.

Fabre, P-H, L Hautier, D Dimitrov, EJP Douzery. 2012. A glimpse on the pattern of rodent diversification: a phylogenetic approach. Bmc Evolutionary Biology 12.

Felsenstein, J. 1985. Phylogenies and the comparative method. American Naturalist 125:1-15.

Fernandez, MH, ES Vrba. 2005. A complete estimate of the phylogenetic relationships in Ruminantia: a dated species-level supertree of the extant ruminants. Biological Reviews 80:269-302.

Fitch, WM. 1983. Does the molecular clock keep time? Heredity 50:213.

Gillooly, JF, AP Allen, GB West, JH Brown. 2005. The rate of DNA evolution: effects of body size and temperature on the molecular clock. Proc. Natl. Acad. Sci. USA 102:140-145.

Giraud, M, I Matic, O Tenaillon, M Radman, M Fons, F Taddei. 2001. Costs and benefits of high mutation rates: adaptive evolution of bacteria in the mouse gut. Science 291:2606-2608.

Hartl, DL. 1988. A primer of population genetics. Sunderland, Massachusetts: Sinauer.

Jin, L, C Macaubas, J Hallmayer, A Kimura, E Mignot. 1996. Mutation rate varies among alleles at a microsatellite locus: phylogenetic evidence. Proc. Natl. Acad. Sci. USA 93:15285-15288. 
482

483

484

485

486

487

488

489

490

491

492

493

494

495

496

497

498

499

500

501

502

503

504

505

506

507

508

509

510

511

512

513

514

515

516

517

518

519

520

521

522

523

524

525

526

527

528

529

Kelkar, YD, S Tyekucheva, F Chlaromonte, K Makova. 2008. The genome-wide determinants of human and chimpanzee microsatellite evolution. Genome Res. 18:30-38.

Khaidakov, M, ER Siegel, RJ Shmookler Reis. 2006. Direct repeats in mitochondrial DNA and mammalian lifespan. Mechanisms Ageing Dev. 127:808-812.

Lanfaer, R, JJ Welch, L Bromham. 2010. Watching the clock: studying variation in rates of molecular evolution between species. Trends Ecol. Evol. 25:495-503.

Langergraber, KE, K Pruefer, C Rowney, C Boesch, C Crockford, K Fawcett, E Inoue, M Inoue-Muruyama, JC Mitani, MN Muller, MM Robbins, G Schubert, TS Stoinski, B Viola, D Watts, RM Wittig, RW Wrangham, K Zuberbuehler, S Paeaebo, L Vigilant. 2012. Generation times in wild chimpanzees and gorillas suggest earlier divergence times in great ape and human evolution. Proc. Natl. Acad. Sci. USA 109:15716-15721.

Lartillot, N, R Poujol. 2011. A phylogenetic model for investigating correlated evolution of substitution rates and continuous phenotypic characters. Mol. Biol. Evol. 28:729-744.

Margoliash, E. 1963. Primary structure and evolution of cytochrome C. Proc. Natl. Acad. Sci. USA 50:672-679.

Martin, AP, SR Palumbi. 1993. Body size, metabolic rate, generation time, and the molecular clock. Proc. Natl. Acad. Sci. USA 90:4087-4091.

Masters, BS, LS Johnson, BGP Johnson, JL Brubaker, SK Sakaluk, CF Thompson. 2011. Evidence for heterozygote instability in microsatellite loci in house wrens. Biol. Lett. 7:127-130.

Meredith, RW, JE Janecka, J Gatesy, OA Ryder, CA Fisher, EC Teeling, A Goodbla, E Eizirik, TLL Simao, T Stadler, DL Rabosky, RL Honeycutt, JJ Flynn, CM Ingram, C Steiner, TL Williams, TJ Robinson, A Burk-Herrick, M Westerman, NA Ayoub, MS Springer, WJ Murphy. 2011. Impacts of the Cretaceous Terrestrial Revolution and KPg Extinction on Mammal Diversification. Science 334:521-524.

Messier, W, S-H Li, C-B Stewart. 1996. The birth of microsatellites. Nature 381:483.

Nabholz, B, S Glémin, N Galtier. 2009. The erratic mitochondrial clock: variations in mutation rate, not population size, affect mtDNA diversity across birds and mammals. Bmc Evolutionary Biology 9:54.

Ohta, T. 1987. Very slightly deleterious mutations and the molecular clock. J. Mol. Evol. 26:1-6.

Osada, N, K Hashimoto, Y Kameoka, M Hirata, R Tanuma, Y Uno, I Inoue, M Hida, Y Suzuki, S Sugano, K Terao, J Kusuda, I Takahashi. 2008. Largescale analysis of Macaca fascicularis transcripts and inference of genetic divergence between M-fascicularis and M-mulatta. BMC Genomics 9.

Pacifici, M, L Santini, M Di Marco, D Baisero, L Francucci, GG Marasini, P Visconti, C Rondinini. 2013. Generation length for mammals. Nature Cons. 5:89-94.

Paradis, E, J Claude, K Strimmer. 2004. APE: Analysis of Phylogenetics and Evolution in R language. Bioinformatics 20:289-290. 
530

531

532

533

534

535

536

537

538

539

540

541

542

543

544

545

546

547

548

549

550

551

552

553

554

555

556

557

558

559

560

561

562

563

564

565

566

567

568

569

570

571
Perelman, P, WE Johnson, C Roos, HN Seuanez, JE Horvath, MAM Moreira, B Kessing, J Pontius, M Roelke, Y Rumpler, MPC Schneider, A Silva, SJ O'Brien, J Pecon-Slattery. 2011. A Molecular Phylogeny of Living Primates. PLoS genetics 7:e1001342, 1001341-1001317.

Primmer, C, H Ellegren, N Saino, AP Møller. 1996. Directional evolution in germline microsatellite mutations. Nature Genetics 13:391-393.

Purvis, A, A Rambaut. 1995. Comparative analysis by independent contrasts (CAIC): an Apple Macintosh application for analysing comparative data. CABIOS 11:247-251.

Samuels, DC. 2004. Mitochondrial DNA repeats constrain the life span of mammals. Trends Genet. 20:226-229.

Schlötterer, C, D Tautz. 1992. Slippage synthesis of simple sequence DNA. Nucleic Acids Research 20:211-215.

Schrider, DR, JN Hourmozdi, MW Hahn. 2011. Pervasive multinucleotide mutation events in eukaryotes. Curr. Biol. 21:1051-1054.

Slatkin, M. 1995. A measure of population subdivision based on microsatellite allele frequencies. Genetics 139:457-462.

Smith, SA, MJ Donoghue. 2008. Rates of molecular evolution are linked to life history in flowering plants. Science 322:86-89.

Sun, JX, A Helgason, G Masson, SS Ebenesersdottir, H Li, S Mallick, S Gnerre, N Patterson, A Kong, D Reich, K Stefansson. 2012. A direct characterization of human mutation based on microsatellites. Nature Genetics 44:1161-+.

Taylor, JS, MH Durkin, F Breden. 1999. The death of a microsatellite: a phylogenetic perspective on microsatellite interruptions. Mol. Biol. Evol. 16:567-572.

The Huntington's Disease Collaborative Research Group. 1993. A novel gene containing a trinucleotide repeat that is expanded and unstable on Huntington's disease chromosomes. Cell 72:971-983.

Tuntiwechapikul, W, M Satazar. 2002. Mechanism of in vitro expansion of long DNA repeats: effect of temperature, repeat length, repeat sequence and DNA polymerases. Biochemistry 41:854-860.

Weber, JL. 1990. Informativeness of human (dC-dA)n . (dG-dT)n polymorphisms. Genomics 7:524-530.

Welch, JJ, ORP Bininda-Emonds, L Bromham. 2008. Correlates of substitution rate variation in mammalian protein-coding sequences. Bmc Evolutionary Biology 8:53.

$\mathrm{Xu}, \mathrm{X}, \mathrm{M}$ Peng, Z Fang, $\mathrm{X} \mathrm{Xu}$. 2000. The direction of microsatellite mutations is dependent upon allele length. Nat. Genet. 24:396-399.

Zuckerkandl, E, LE Pauling. 1962. Molecular disease, evolution, and genic heterogeneity. In: M Kasha, B Pullman, editors. Horizons in Biochemistry. New York: Academic Press. p. 189-225. 


\section{Figure 1}

Variation in relative frequency of different length $\mathrm{AC}$ microsatellites in six higher primates.

The six higher primates are: human (HAS, black), bonobo (PPA, blue), chimpanzee (PTR, red), gorilla (GGO, pink), orang-utan (PAB, green) and gibbon (NLE, yellow). All frequencies are expressed as natural logarithms and individual data points are then calculated as deviations from the group mean. A value of one thus indicates a frequency 2.7 times the geometric mean of the six species.

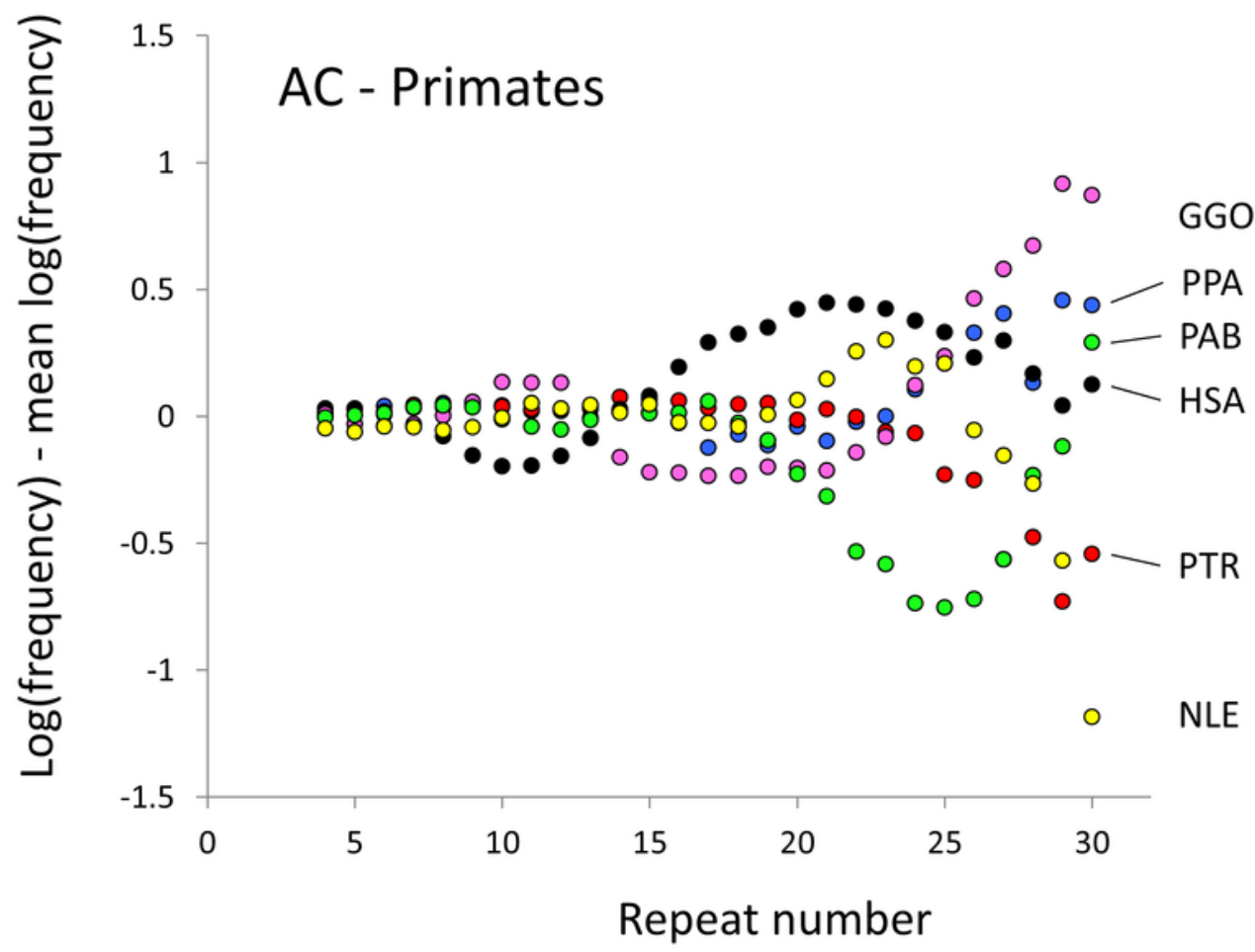


Figure 2

Variation in relative frequency of different length AT microsatellites in higher primates

For details, see legend Figure 1.

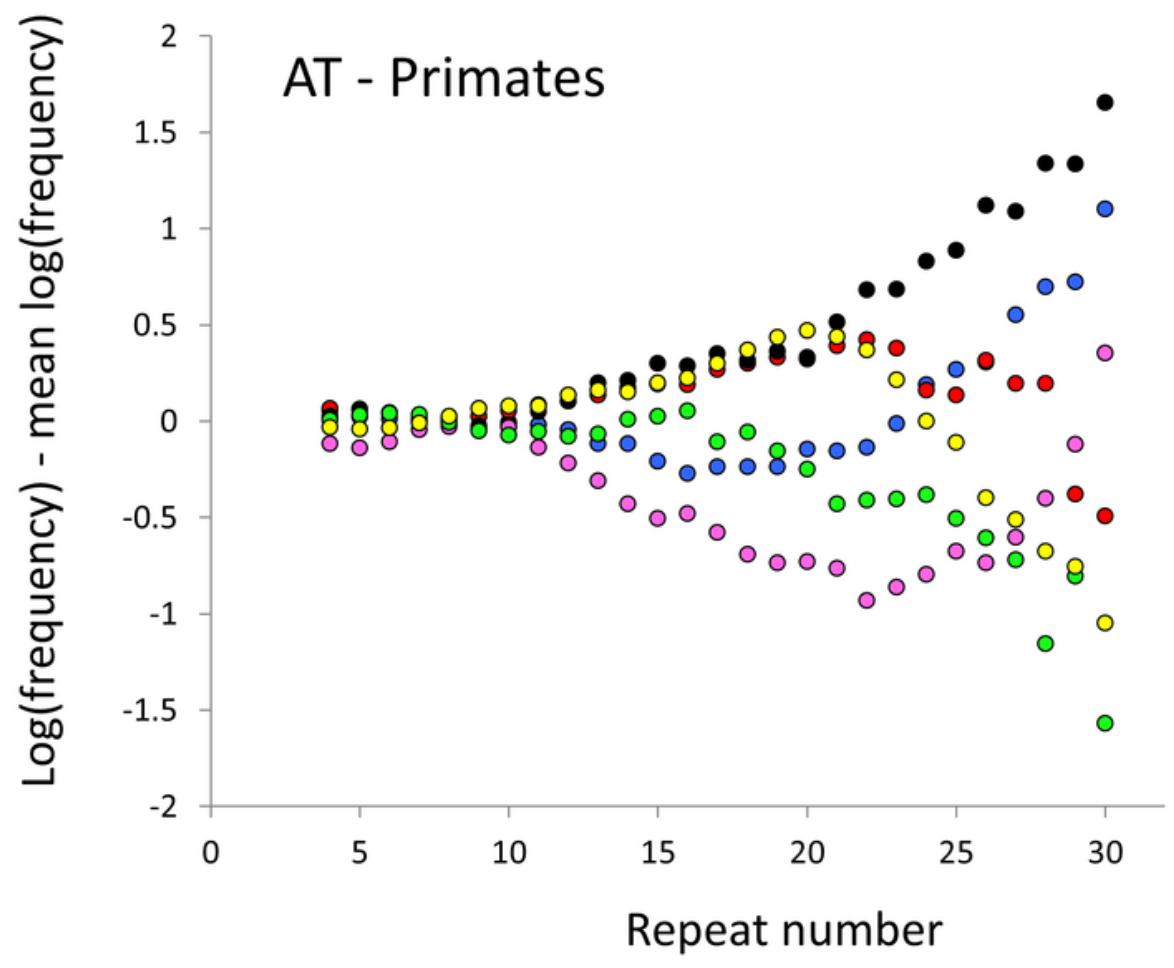




\section{Figure 3}

Variation in relative frequency of different length AC microsatellites in seven Afrotheria The seven Afrotheria are: hyrax (PCA, green), tenrec (ETE, black), manatee (TMA, light blue), elephant shrew (EED, pink), golden mole (CAS, blue), elephant (LAF, red) and aardvark (OAF, yellow). All frequencies are expressed as natural logarithms and individual data points are then calculated as deviations from the group mean. A value of one thus indicates a frequency 2.7 times the geometric mean of the seven species.

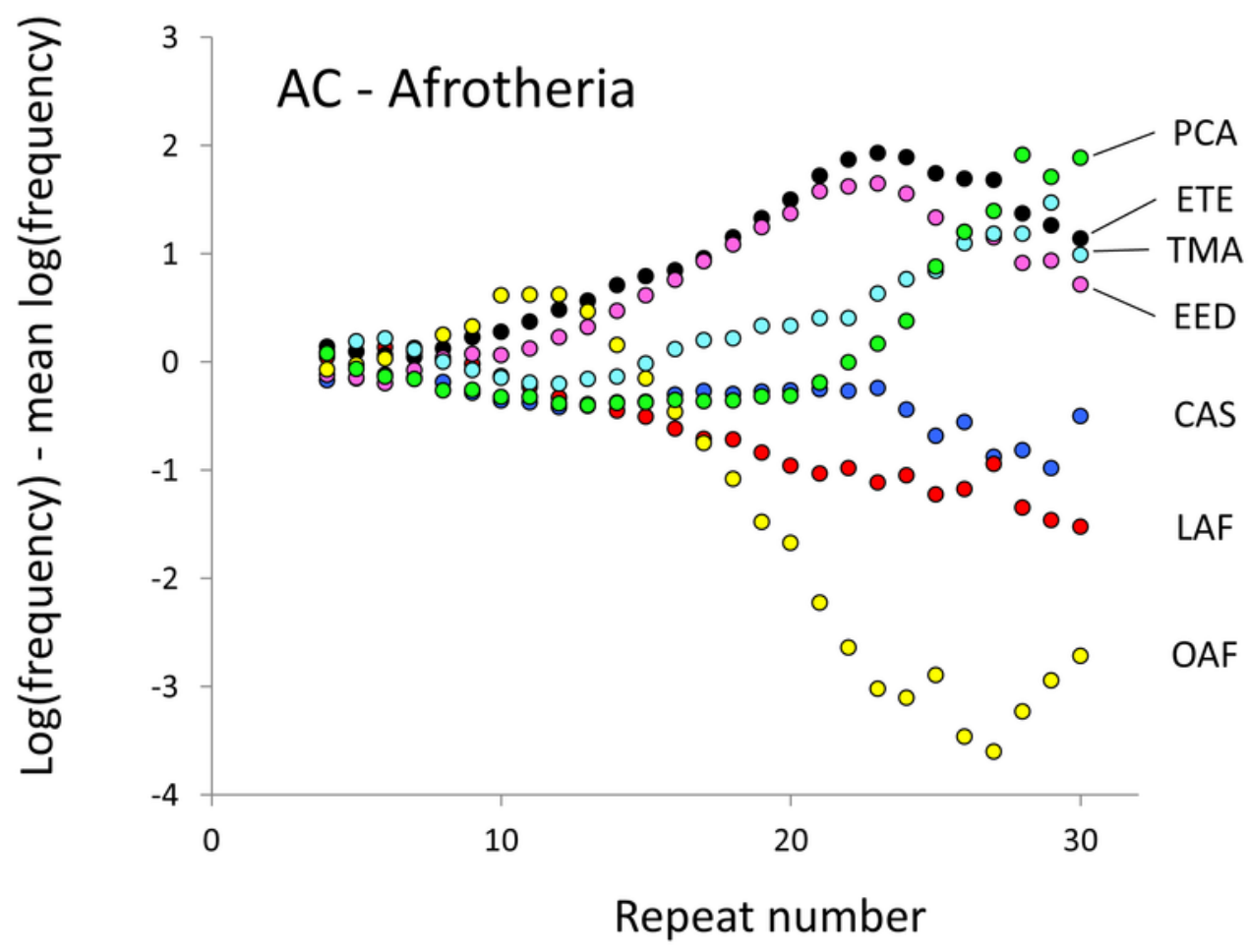


Figure 4

Variation in relative frequency of different length AT microsatellites in seven Afrotheria

For details, see legend Figure 3.

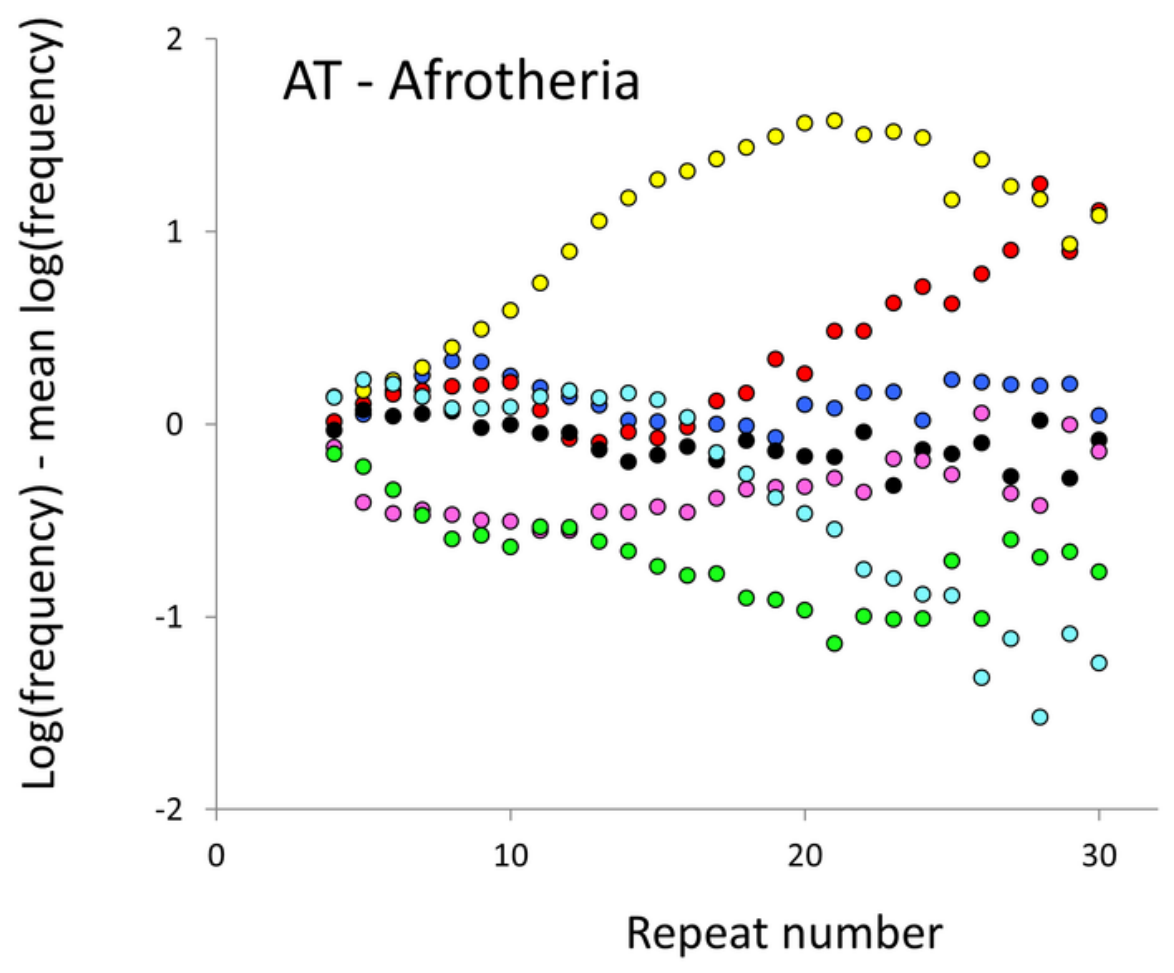


Figure 5

Relationship between body temperature and microsatellite frequency.

Three microsatellite motifs were analysed $(\mathrm{AT}, \mathrm{AC}$ and $\mathrm{AG}=$ black, grey and white data series respectively). The vertical axis is the estimate of the slope of the relationship between body temperature and $\log _{\mathrm{e}}$ microsatellite frequency, corrected for body mass and the genome's GC\%. All variables are included as phylogenetically independent contrasts. Three data points with high leverage were omitted. Error bars are one standard error of the estimate.

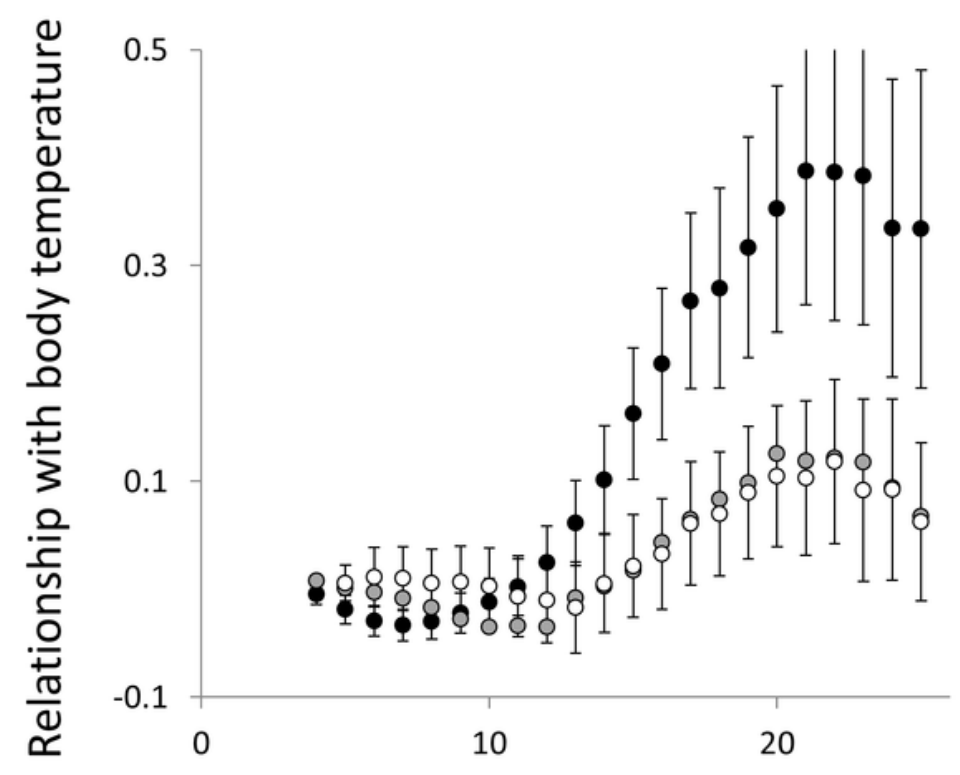

Repeat units 


\section{Figure 6}

Relationship between body mass and microsatellite frequency.

Three microsatellite motifs were analysed (AT, AC and AG = black, grey and white data series respectively). The vertical axis is the estimate of the slope of the relationship between body mass and loge microsatellite frequency, corrected for body temperature and the genome's GC\%. All variables are included as phylogenetically independent contrasts. Three data points with high leverage, all corresponding to deeper nodes, were omitted. Error bars are one standard error of the estimate.

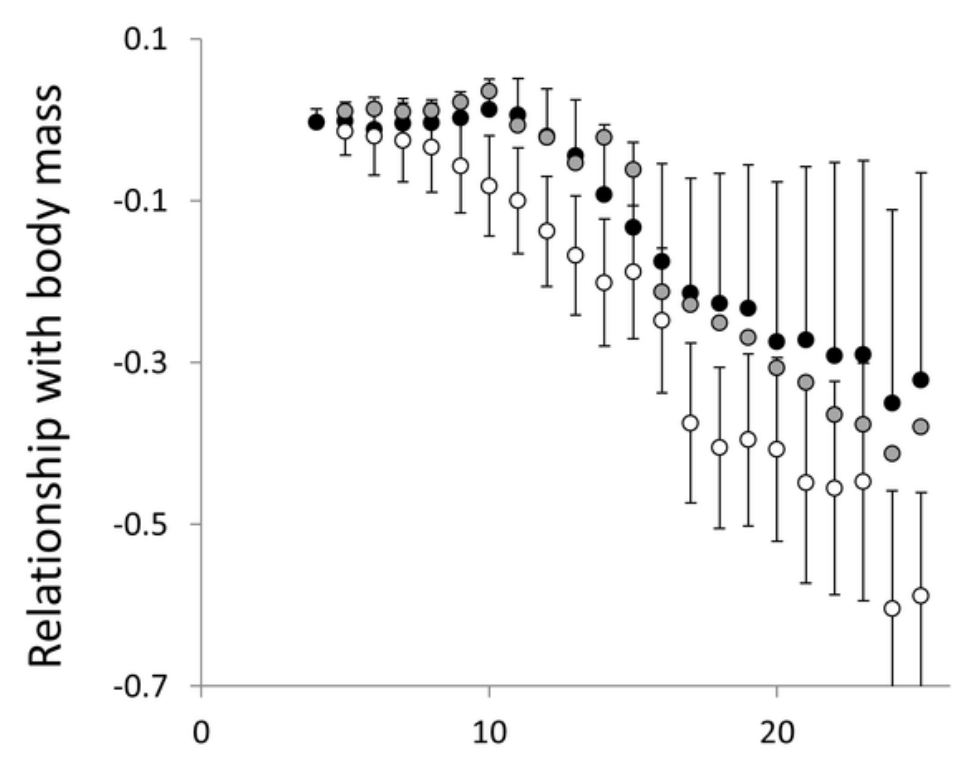

Repeat units 
Figure 7

Relationship between body temperature and the frequency in interrupted microsatellites.

The analysis presented in Figure 5 is repeated, but this time using microsatellites carrying a single interruption (for full definition see text).

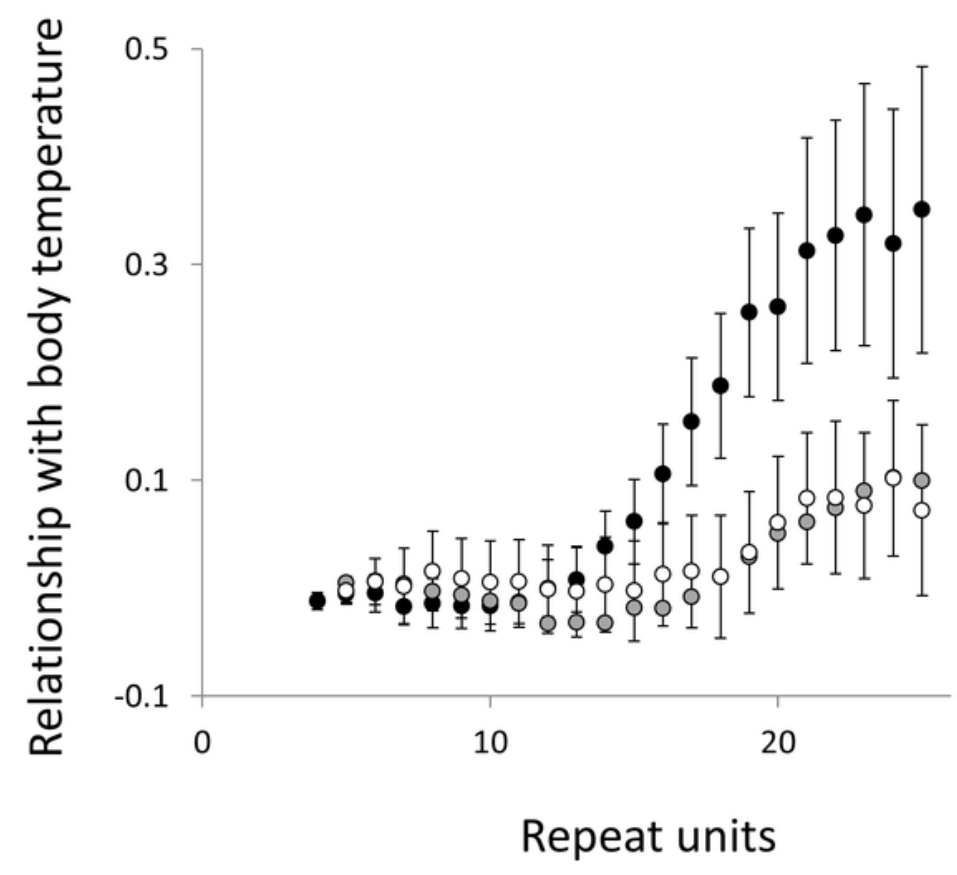


Figure 8

Relationship between body mass and the frequency in interrupted microsatellites.

The analysis presented in Figure 6 is repeated, but this time using microsatellites carrying a single interruption (for full definition see text).

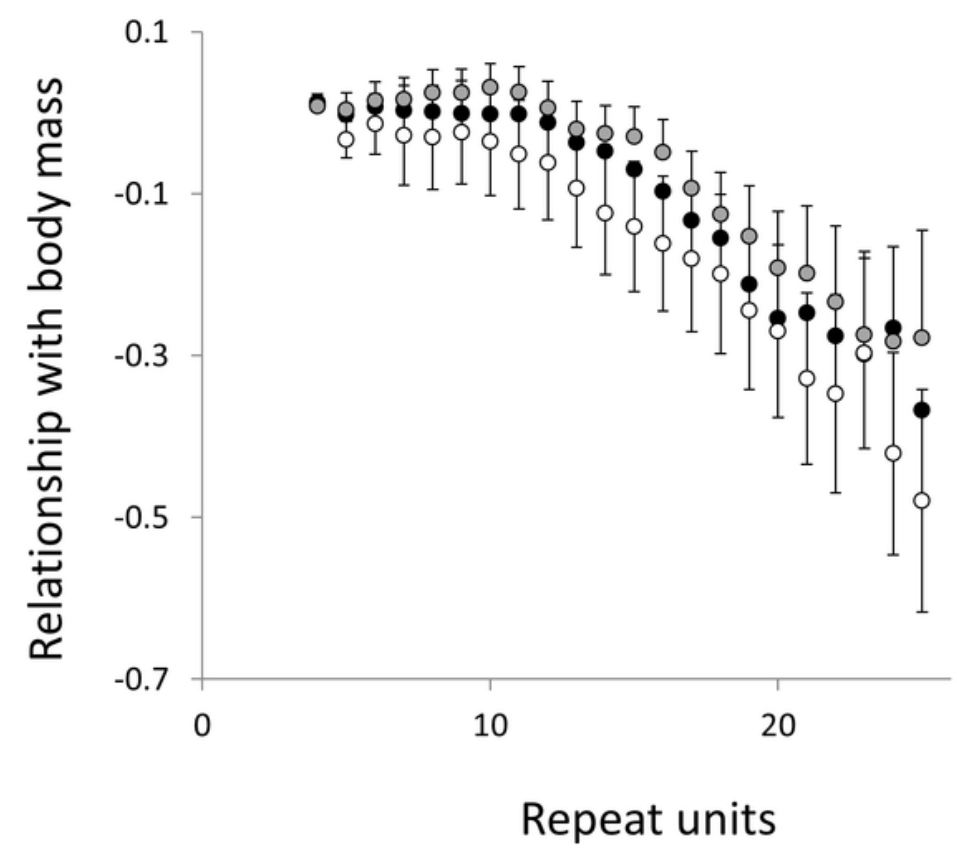


Figure 9

Using microsatellites to predict body weight.

All data are expressed as $\log _{10}$ (mass in kilograms). Horizontal axis is actual adult body weight. Vertical axis is body weight predicted from the frequencies of microsatellites in that species' genome, using simple multiple regressions based on all species not in the same major clade. The arrow indicates the killer whale, a large outlier.

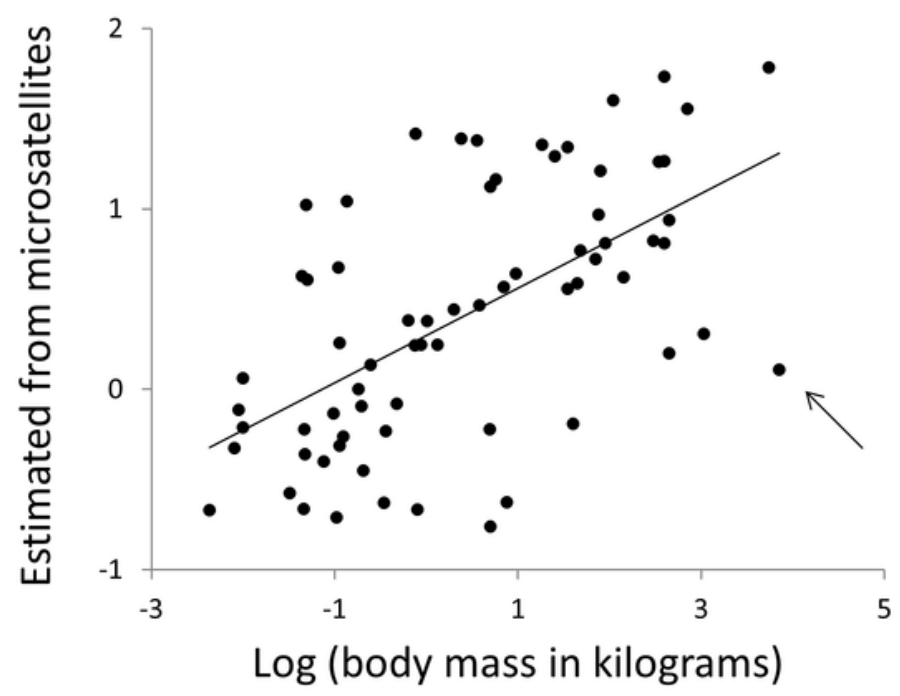

